\title{
Gengnianchun, a Traditional Chinese Medicine, Enhances Oxidative Stress Resistance and Lifespan in Caenorhabditis elegans by Modulating daf-16/FOXO
}

\author{
Fanhui Meng, ${ }^{1,2}$ Jun Li, ${ }^{2}$ Wenjun Wang, ${ }^{2}$ and Yan $\mathrm{Fu}^{1}$ \\ ${ }^{1}$ Department of Gynecology, The First Hospital of Jilin University, Changchun, China \\ ${ }^{2}$ Department of Integrated Traditional Chinese Medicine and Western Medicine, Obstetrical and Gynecological Hospital, \\ Fudan University, Shanghai, China \\ Correspondence should be addressed to Wenjun Wang; wenjunwang63@163.com and Yan Fu; f_y@jlu.edu.cn
}

Received 5 November 2016; Revised 24 February 2017; Accepted 6 March 2017; Published 16 March 2017

Academic Editor: Hyunsu Bae

Copyright (C) 2017 Fanhui Meng et al. This is an open access article distributed under the Creative Commons Attribution License, which permits unrestricted use, distribution, and reproduction in any medium, provided the original work is properly cited.

\begin{abstract}
Objective. Gengnianchun (GNC), a traditional Chinese medicine (TCM), is primarily used to improve declining functions related to aging. In this study, we investigated its prolongevity and stress resistance properties and explored the associated regulatory mechanism using a Caenorhabditis elegans model. Methods. Wild-type C. elegans N2 was used for lifespan analysis and oxidative stress resistance assays. Transgenic animals were used to investigate pathways associated with antioxidative stress activity. The effects of GNC on levels of reactive oxygen species (ROS) and expression of specific genes were examined. Results. GNC-treated wild-type worms showed an increase in survival time under both normal and oxidative stress conditions. GNC decreased intracellular ROS levels by $67.95 \%$. GNC significantly enhanced the oxidative stress resistance of several mutant strains, suggesting that the protective effect of GNC is independent of the function of these genes. However, the oxidative stress resistance effect of GNC was absent in worms with daf-16 mutation. We also found upregulation of daf-16 downstream targets including sod-3 and mtl-1. Conclusions. Our findings suggest that GNC extends the lifespan of C. elegans and enhances its resistance to oxidative stress via a daf-16/FOXOdependent pathway. This study also provides a feasible method for screening the biological mechanisms of TCMs.
\end{abstract}

\section{Introduction}

Gengnianchun (GNC) is a traditional Chinese medicine (TCM) composed of 12 ingredients: Radix Rehmanniae, Rhizoma Coptidis, Radix Paeoniae Alba, Rhizoma Anemarrhenae, Cistanche salsa, Radix Morindae Officinalis, Poria, Epimedium brevicornum, Cortex Phellodendri Amurensis, Fructus lycii, Semen Cuscutae, and Carapax et Plastrum Testudinis. According to TCM theory, GNC has a kidney/livertonifying effect that is used to alleviate declining functions related to aging. Moreover, according to clinical data, GNC has therapeutic effects on menopausal symptoms [1]. Recently, numerous studies have indicated that GNC provides an array of beneficial effects. For example, GNC improves learning and memory in ovariectomized rats by increasing hippocampal acetylcholine (ACh), acetylcholinesterase (AChE), and choline acetyltransferase (ChAT) activities [2]. Serum of GNC-treated rats inhibits apoptosis in $\mathrm{A} \beta$-insulted $\mathrm{PC}-12$ cells by regulating $\mathrm{Bcl}-2$ and $\mathrm{Bax}$ expression and caspase 3 activation. These effects suggest that GNC may protect against neurodegenerative diseases such as Alzheimer's disease [3]. Furthermore, GNC exerts antiaging effects by modulating the hypothalamus-pituitaryovary axis, thereby increasing the level of estradiol receptor (ER) in the pituitary gland and ovaries as well as increasing the concentration of $\beta$-EP in the hypothalamus [4]. More recently, Rao et al. demonstrated that GNC decreased levels of interleukin-1 beta (IL-1 $\beta$ ), interleukin-6 (IL-6), and tumor necrosis factor-alpha (TNF- $\alpha$ ) but increased levels of interleukin-2 (IL-2) and interferon-gamma (IFN- $\gamma$ ). Using an ovariectomized rat model, GNC was also found to reverse ovariectomy-induced weight gain and leptin resistance, with possible clues regarding the mechanisms through which GNC attenuates age-related diseases [5]. Using a skin-aging 
TABLE 1: Composition and preparation of GNC.

\begin{tabular}{lcc}
\hline TCM ID & Crude herb $(\mathrm{g})$ & Water extract $(\mathrm{g})$ \\
\hline Radix Rehmanniae & 15 & 4.5 \\
Rhizoma Coptidis & 3 & 0.5 \\
Radix Paeoniae Alba & 12 & 1.2 \\
Rhizoma Anemarrhenae & 15 & 3.75 \\
Cistanche salsa & 12 & 3.6 \\
Radix Morindae Officinalis & 12 & 3.6 \\
Poria & 9 & 0.9 \\
Epimedium brevicornum & 12 & 0.6 \\
Cortex Phellodendri Amurensis & 9 & 0.75 \\
Fructus lycii & 12 & 4.8 \\
Semen Cuscutae & 12 & 0.6 \\
Carapax et Plastrum Testudinis & 15 & 0.75 \\
\hline
\end{tabular}

Notes: conversion between crude herb and water extract: for example, $4.5 \mathrm{~g}$ water extract of Radix Rehmanniae is equivalent to $15 \mathrm{~g}$ crude herb.

model, Yuan demonstrated that GNC significantly increases the concentration of hydroxyproline (HYP) and activity of SOD, suggesting that GNC improves antioxidative defense against aging [6].

Oxidative stress represents an imbalance between toxic reactive oxygen species (ROS) and antioxidant systems. High levels of ROS cause inflammation and cytotoxicity, damaging proteins, lipids, and DNA, and subsequently disrupting cellular functions and resulting in cell death [7]. In humans, oxidative stress plays an important role in many diseases, including cardiovascular diseases [8], neurodegenerative diseases such as Alzheimer's disease [9] and Parkinson's disease [10], autoimmune diseases such as SLE [11], and cancer [12]. Furthermore, numerous studies have demonstrated the extensive involvement of oxidative stress in aging itself $[13,14]$.

Caenorhabditis elegans is a powerful animal model because of its short lifespan, ability to self-fertilize, and ease of culture. Because it possesses genes homologous to two-thirds of those involved in human disease, C. elegans is applied as a well-characterized experimental system for studying aging and aging-associated diseases [15], and several studies have shown that enhanced resistance to oxidative stress extends the lifespan of this nematode $[16,17]$. In C. elegans, stress resistance is related to many factors, including insulin/IGF1 , TOR, and mitochondrial signaling pathways [18-20]. In addition, a rich collection of mutants has been used to explore the molecular mechanisms of the therapeutic components of TCM pharmacopeia, such as Liuwei Dihuang (LWDH) and Aiweixin [21, 22].

To investigate whether the TCM GNC exhibits stress resistance properties, the potential to extend longevity, or both, in vivo lifespan and antioxidant assays were performed. This study also utilized wild-type and mutant C. elegans strains to determine associated regulatory mechanisms of the oxidative stress resistance induced by GNC, and the effects of GNC on the expression levels of associated genes were analyzed.

\section{Materials and Methods}

2.1. Preparation of the GNC Formula. The GNC formula used contains 12 crude herbs (as shown in Table 1). For this study, we used a mixture of water extracts of the 12 crude herbs. The water extracts, which were purchased from Tianjiang Pharmaceutical (Jiangyin, China), were produced following the rigid specifications of the Pharmacopeia of the People's Republic of China and were CFDA-approved. The conversion between crude herb and water extract is also shown in Table 1. According to conventional TCM research, all concentrations in this study referred to the concentration of the crude herb.

2.2. C. elegans: Strains and Maintenance. The Caenorhabditis Genetics Center (CGC) at the University of Minnesota (Minneapolis, MN, USA) provided wild-type C. elegans N2 (Bristol), E. coli OP50, and the following transgenic worms: CB1370, daf-2 (e1370); TJ1052, age-1 (hx546); CF1038, daf16 (mu86); MQ1333, nuo-6 (qm200); MQ887, isp-1 (qm150); DA465, eat-2 (ad465); RB1206, rsks-1 (ok1255) and CF1903, glp-1 (e2144). C. elegans strains were maintained at $20^{\circ} \mathrm{C}$ on solid nematode growth medium (NGM) plates seeded with $E$. coli OP50. For CB1370, daf-2 (e1370) cultures, the nematodes were maintained at $16^{\circ} \mathrm{C}$ for 3 days and then transferred to $20^{\circ} \mathrm{C}$ until the desired stage of development was reached.

2.3. Toxicity Test for GNC. The toxic effect of different concentrations of GNC was evaluated. Age-synchronized day 1 adult N2 C. elegans were incubated with a series of GNC concentrations, from $0.00394 \mathrm{mg} / \mathrm{mL}$ to $78.8 \mathrm{mg} / \mathrm{mL}$, in Scomplete liquid medium (a liquid culture medium for $C$. elegans) [23]. A final concentration of $400 \mu \mathrm{M} 5$-fluoro- $2^{\prime}$ deoxyuridine (FUDR, Sigma-Aldrich Co., St. Louis, MO, USA) was added to the medium to block progeny development. Survival was assessed after 48 hours of treatment with GNC. The nematodes were considered dead when they failed to respond to touch using a platinum loop. Ninety worms at each concentration were tested, and the experiment was performed three times independently.

2.4. Lifespan Analysis. Age-synchronized day 1 adult N2 nematodes were transferred to a 96-well plate with 1-2 worms in $80 \mu \mathrm{L}$ of S-complete liquid medium containing various GNC drug concentrations or a vehicle control $\left(\mathrm{H}_{2} \mathrm{O}\right)$ in each well. E. coli OP50 and FUDR were added to the medium. 
Survival was assessed every other day until death using the touch-provoked method described above. The lifespan assay was repeated in three independent trails.

2.5. Oxidative Stress Resistance Assay. The oxidative stress resistance assay was based on previously published studies $[22,24]$. Briefly, age-synchronized day 1 adult worms were incubated in several concentrations of GNC $(0.0394,0.394$, $1.97,3.94$, and $11.82 \mathrm{mg} / \mathrm{mL}$ ) in S-complete liquid medium with FUDR for 48 hours; the worms were then transferred to a 48 -well plate, with approximately 35 worms in $160 \mu \mathrm{L}$ of M9 buffer per well [24]. Oxidative stress was induced with $10 \mathrm{mM}$ hexavalent chromium ( $\mathrm{Cr}$ [VI], $\mathrm{K}_{2} \mathrm{Cr}_{2} \mathrm{O}_{7}$, Sangon Biotech, Shanghai, China); before the stressor was added, the worms were washed three times with M9 buffer to remove OP50 bacteria. Worm survival was monitored regularly every 4 hours using the touch-provoked movement described above. The experiment was conducted in three independent repeats.

2.6. Measurement of ROS Production. The effect of GNC on ROS levels in C. elegans under oxidative stress conditions induced by $\mathrm{Cr}$ (VI) was measured using $\mathrm{H}_{2}$ DCF-DA (SigmaAldrich Co., St. Louis, MO, USA) as a molecular probe. The procedures for GNC and Cr (VI) administration were the same as those outlined above for the oxidative stress resistance assay. The worms were washed three times with M9 buffer, and $50 \mu \mathrm{M} \mathrm{H}_{2}$ DCF-DA was added to each well. After incubating for 3 hours at $20^{\circ} \mathrm{C}$, the worms were mounted onto microscope slides with $2 \%$ agar pads. The worms were anesthetized with $10 \mathrm{mM} \mathrm{NaN}_{3}$ and then analyzed using a Nikon SMZ 1500 fluorescence microscope at 480/40 nm excitation and 535/50 nm emission. Quantitative image analyses were performed by image-based morphometric analysis (NISElements D3.1, Japan) and Image J software (US National Institutes of Health, Bethesda, MD, US). Inverted fluorescent images were used for the analysis. Positive signals were defined with Image J. At least 20 animals from each group were quantified, and the experiment was performed three times independently.

2.7. Quantitative Analysis of Stress-Induced Genes in C. elegans. Synchronized adult worms were exposed to $\mathrm{Cr}$ (VI) as described above for 15 hours at $20^{\circ} \mathrm{C}$. The worms were collected, washed three times with M9 buffer, transferred to RNase-free microfuge tubes, and pelleted by centrifugation at 4,000 rpm for $1 \mathrm{~min}$. The samples were freeze-thawed, and $1 \mathrm{~mL}$ TRIzol reagent (Thermo Fisher Scientific, Shanghai, China) was added to each sample. For RNA extraction, $200 \mu \mathrm{L}$ of chloroform was added, and the worm suspension was shaken vigorously and centrifuged at $12,000 \times \mathrm{g}$ for $10 \mathrm{~min}$. The total nematode RNA in the supernatant was isolated using isopropanol and washed with ethanol. The RNA concentration was quantified using a Nanodrop spectrophotometer. cDNA was synthesized by reverse transcription using FastQuant RT Kit (with gDNase; Tiangen, Beijing, China) according to the manufacturer's protocol. Quantitative real-time polymerase chain reaction (qPCR) was performed using qTOWER 2.2 Real-Time PCR System
(Analytik Jena AG, Thuringia, Germany) with SuperReal PreMix Plus (SYBR Green; Tiangen, Beijing, China). The primers were as follows: sod-3, forward, $5^{\prime}$-AGCATCATGCCACCTACGTGA-3', and reverse, $5^{\prime}$-CACCACCATTGAATTTCAGCG-3'; ctl-2, forward, $5^{\prime}$-TCCGTGACCCTATCCACTTC-3', and reverse, $5^{\prime}$-TGGGATCCGTATCCATTCAT-3'; mtl-1, forward, $5^{\prime}$-ATGGCTTGCAAGTGTGACTGCAAAAACAAGC- $3^{\prime}$, and reverse, 5' ${ }^{\prime}$ TTAATGAGCCGCAGCAGTTCCCTGGTGTTGATGGG-3'; hsp-12.6, forward, $5^{\prime}$ GTGATGGCTGACGAAGGAAC-3', and reverse, $5^{\prime}$-GGGAGGAAGTTATGGGCTTC-3'; and hsp-16.2, forward, $5^{\prime}$ CTGCAGAATCTCTCCATCTGAGTC-3', and reverse, $5^{\prime}$ AGATTCGAAGCAACTGCACC-3'. The gene act-4 (forward: $5^{\prime}$-GCCACCGCTGCCTCCTCATC- $3^{\prime}$ and reverse: $5^{\prime}$ CCGGCAGACTCCATACCCAAGAAG- ${ }^{\prime}$ ) was used as a nonvariable control. Relative fold changes were calculated using the $2^{-\Delta \Delta C T}$ method. The experiment was repeated in triplicate.

2.8. Statistical Analyses. GraphPad Prism 6.0 was used for statistical analyses. For the lifespan assay, Kaplan-Meier survival analysis was conducted, and $p$ values were calculated using the log-rank test. Student's $t$-test was performed to compare two datasets. One-way analysis of variance (ANOVA) with Duncan's post hoc test was used to compare more than two datasets. All results were expressed as the means \pm standard error of the mean (SEM). Values of $p<$ 0.05 were considered significant.

\section{Results}

3.1. GNC Extended Wild-Type C. elegans N2 Lifespan under Normal Conditions. To evaluate whether GNC has prolongevity properties under normal conditions, we treated wild-type $C$. elegans $\mathrm{N} 2$ on the first day of adulthood with different concentrations of GNC at $20^{\circ} \mathrm{C}$ (we chose GNC doses of $0.0394,0.394,1.97,3.94$, or $7.88 \mathrm{mg} / \mathrm{mL}$, which were found to be nontoxic to the worms (Supplementary Table 1 and Figure 1 in Supplementary Material available online at https://doi.org/10.1155/2017/8432306)). As shown in Figure 1, most doses $(0.394,1.97,3.94$, and $7.88 \mathrm{mg} / \mathrm{mL})$ of GNC significantly increased the mean lifespan of adult worms $(10.0 \%$ for $0.394 \mathrm{mg} / \mathrm{mL}, p=0.0009 ; 21.0 \%$ for $1.97 \mathrm{mg} / \mathrm{mL}, p<0.0001$; $31.3 \%$ for $3.94 \mathrm{mg} / \mathrm{mL}, p<0.0001$; and $23.0 \%$ for $7.88 \mathrm{mg} / \mathrm{mL}$, $p<0.0001)$ compared with the control. However, the dose of $0.0394 \mathrm{mg} / \mathrm{mL}$ did not lead to a significant extension of lifespan $(p=0.7742)$. These results show that $3.94 \mathrm{mg} / \mathrm{mL}$ provided the maximum lifespan increase.

3.2. GNC Treatment Increased Stress Resistance and Reduced ROS Levels under Oxidative Stress Conditions in C. elegans. Numerous studies on $C$. elegans have suggested that enhanced stress resistance is an important factor associated with extending lifespan. To investigate whether GNC has an antioxidative effect, $C$. elegans $\mathrm{N} 2$ adults were pretreated with GNC $(0.0394,0.394,1.97,3.94$, or $7.88 \mathrm{mg} / \mathrm{mL})$ for 48 hours, followed by exposure to $\mathrm{Cr}$ (VI). Cr (VI) is a heavy metal with lethal toxicity that generates intracellular ROS, inducing 
TABLE 2: Survival time data under oxidative stress: effects of GNC treatment on wild-type and mutant C. elegans.

\begin{tabular}{|c|c|c|c|c|c|}
\hline Strain & Treatment $(\mathrm{mg} / \mathrm{mL})$ & Mean survival hours ( \pm SEM) & Worms $(N)$ & $\%$ change & $p$ value \\
\hline \multirow{2}{*}{$\mathrm{N} 2$} & Control & $25.83 \pm 0.71$ & 92 & \multirow{2}{*}{67} & \multirow{2}{*}{$<0.0001$} \\
\hline & 3.94-GNC & $43.13 \pm 1.17$ & 90 & & \\
\hline \multirow{2}{*}{ eat-2 (ad465) } & Control & $35.69 \pm 0.68$ & 89 & \multirow{2}{*}{32} & \multirow{2}{*}{$<0.0001$} \\
\hline & 3.94-GNC & $46.95 \pm 0.90$ & 91 & & \\
\hline \multirow{2}{*}{ rsks-1 (ok1255) } & Control & $37.39 \pm 0.69$ & 98 & \multirow{2}{*}{39} & \multirow{2}{*}{$<0.0001$} \\
\hline & 3.94-GNC & $51.88 \pm 1.02$ & 99 & & \\
\hline \multirow{2}{*}{ isp-1 (qm150) } & Control & $20.40 \pm 0.71$ & 90 & \multirow{2}{*}{42} & \multirow{2}{*}{$<0.0001$} \\
\hline & 3.94-GNC & $28.94 \pm 0.72$ & 94 & & \\
\hline \multirow{2}{*}{ nuo-6 (qm200) } & Control & $19.09 \pm 0.57$ & 92 & \multirow{2}{*}{47} & \multirow{2}{*}{$<0.0001$} \\
\hline & 3.94-GNC & $28.04 \pm 0.72$ & 98 & & \\
\hline \multirow{2}{*}{ glp-1 (e2144) } & Control & $30.54 \pm 0.62$ & 95 & \multirow{2}{*}{24} & \multirow{2}{*}{$<0.0001$} \\
\hline & 3.94-GNC & $37.92 \pm 0.76$ & 96 & & \\
\hline \multirow{2}{*}{ daf-16 (mu86) } & Control & $19.60 \pm 0.63$ & 90 & \multirow{2}{*}{0.06} & \multirow{2}{*}{0.2311} \\
\hline & 3.94-GNC & $20.74 \pm 0.70$ & 92 & & \\
\hline \multirow{2}{*}{ daf-2 (e1370) } & Control & $51.43 \pm 1.56$ & 91 & \multirow{2}{*}{28} & \multirow{2}{*}{$<0.0001$} \\
\hline & 3.94-GNC & $65.87 \pm 1.76$ & 92 & & \\
\hline \multirow{2}{*}{ age-1 (hx546) } & Control & $45.82 \pm 1.31$ & 97 & \multirow{2}{*}{21} & \multirow{2}{*}{$<0.0001$} \\
\hline & 3.94-GNC & $55.61 \pm 1.51$ & 92 & & \\
\hline
\end{tabular}
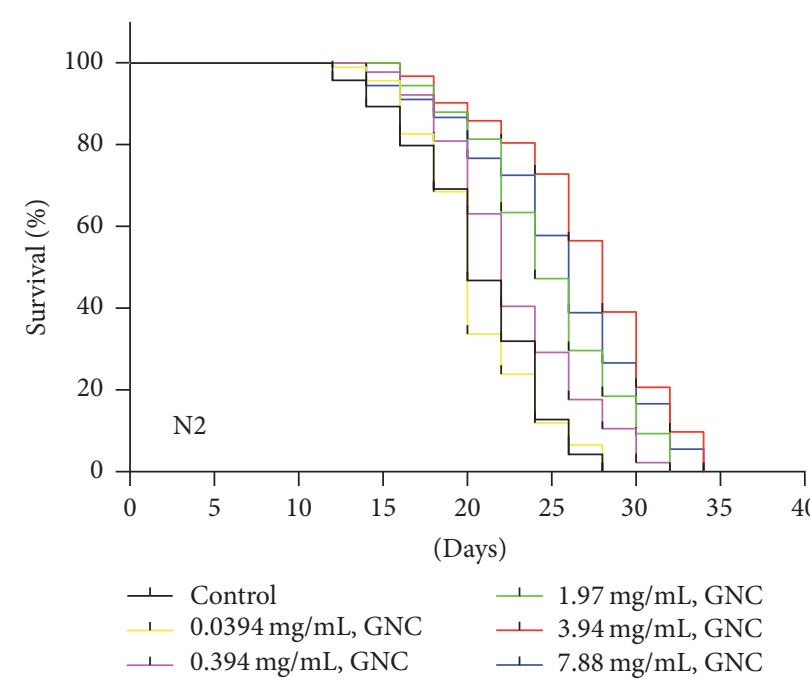

FIGURE 1: Effect of GNC on the lifespan of C. elegans N2 under normal conditions at $20^{\circ} \mathrm{C}$. Notes: the above curves show the survival percentage of worms on different days after treatment with a vehicle control $\left(\mathrm{H}_{2} \mathrm{O}\right)$ or various doses of GNC (0.0394, 0.394, $1.97,3.94$, or $7.88 \mathrm{mg} / \mathrm{mL})$. GNC increased the lifespan of wild-type worms in a dose-dependent manner. The maximum increase in lifespan was observed at a dose of $3.94 \mathrm{mg} / \mathrm{mL} \mathrm{GNC}(p<0.0001)$.

oxidative stress. The results showed that pretreatment with $3.94 \mathrm{mg} / \mathrm{mL}$ GNC maximally increased the mean lifespan of wild-type C. elegans N2 under Cr (VI)-induced oxidative stress by $67.0 \%(43.13 \pm 1.17, p<0.0001)$ compared with the control $(25.83 \pm 0.71$, Table 2$)$. Other doses of GNC pretreatment had a similar antioxidative stress effect (0.394 mg/mL, 21.4\%, $p<0.0001 ; 1.97 \mathrm{mg} / \mathrm{mL}, 32.5 \%, p<$ 0.0001 ; and $7.88 \mathrm{mg} / \mathrm{mL}, 48.9 \%, p<0.0001)$, whereas a

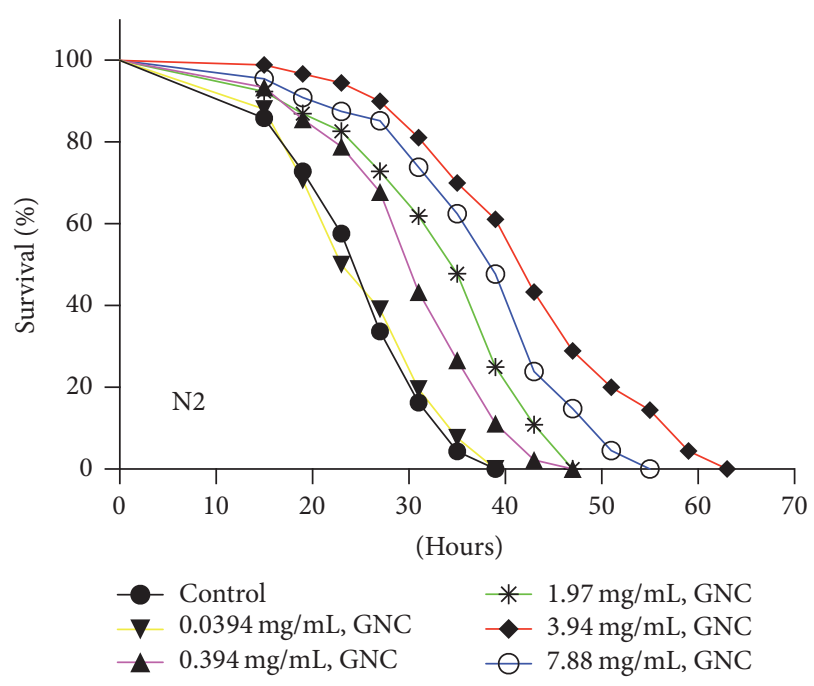

FIGURE 2: The effect of GNC on oxidative stress resistance in C. elegans N2. Notes: GNC $(0.0394,0.394,1.97,3.94$, or $7.88 \mathrm{mg} / \mathrm{mL})$ and vehicle control $\left(\mathrm{H}_{2} \mathrm{O}\right)$-pretreated wild-type worms were exposed to $10 \mathrm{mM}$ Cr (VI). Survival after each treatment was assessed every 4 hours. GNC increased the mean and maximum survival times of worms under oxidative stress conditions in a dose-dependent manner.

lower dose of GNC $(0.0394 \mathrm{mg} / \mathrm{mL}, p=0.8677)$ did not have a significant effect (Figure 2). These findings suggest that GNC enhances stress resistance in a dose-dependent manner. Given that the most significant increase in mean lifespan of wild-type N2 worms under normal or stress conditions was achieved at $3.94 \mathrm{mg} / \mathrm{mL}$ GNC, we chose this concentration to perform ensuing experiments. 


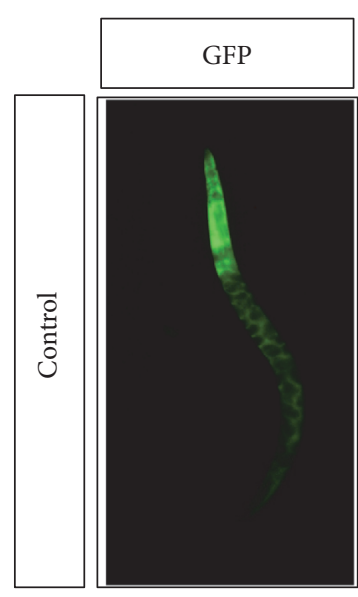

(a)

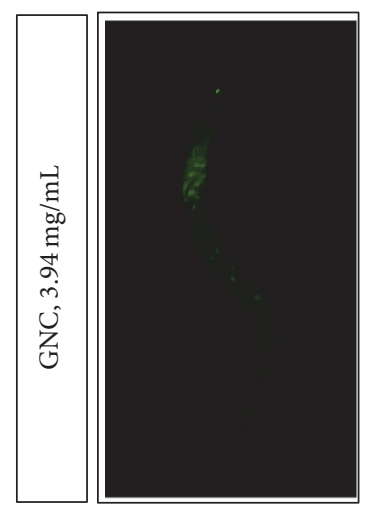

(d)

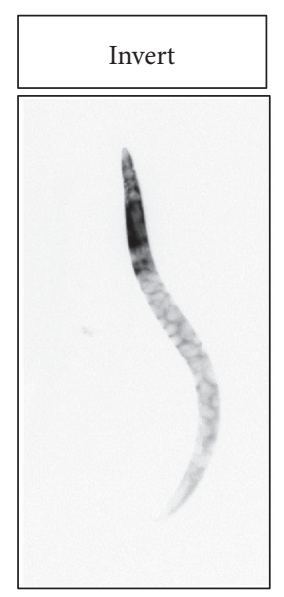

(b)

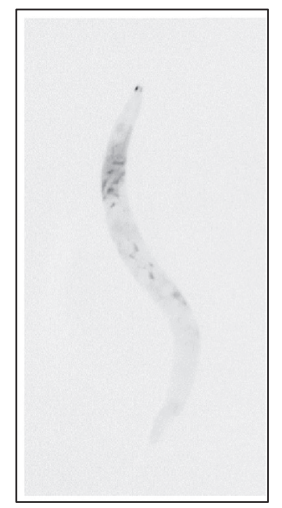

(e)

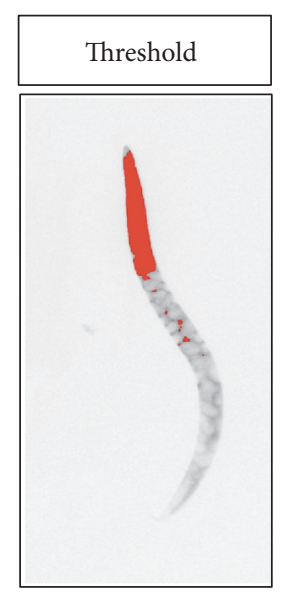

(c)

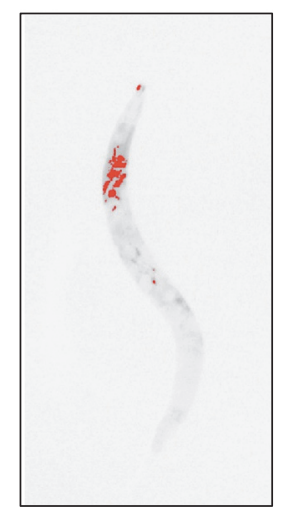

(f)

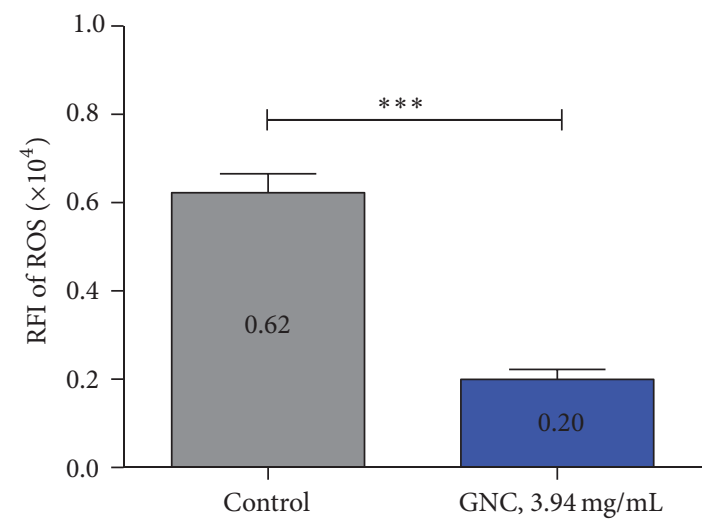

(g)

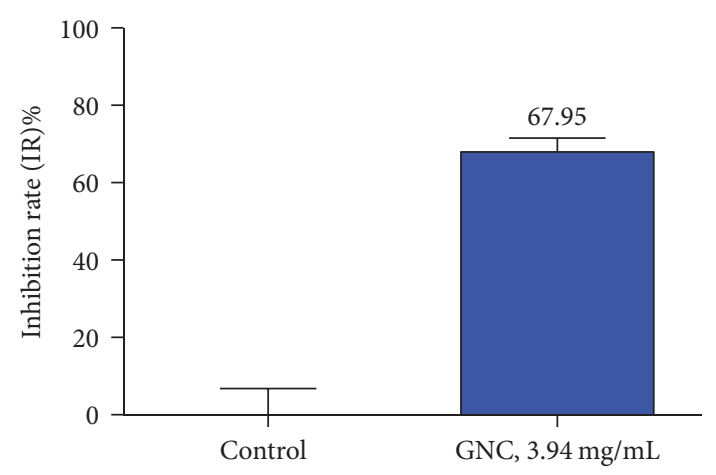

(h)

FIGURE 3: ROS levels of C. elegans under oxidative stress conditions. Notes: worms were exposed to Cr (VI) for 24 hours and stained with the molecular probe $\mathrm{H}_{2}$ DCF-DA. Images of worms pretreated with the vehicle control $\left(\mathrm{H}_{2} \mathrm{O}\right)$ are displayed as follows: fluorescent image (a), inverted image (b), and positive signal (c). Worms pretreated with $3.94 \mathrm{mg} / \mathrm{mL}$ of GNC are displayed as follows: fluorescent image (d), inverted image (e), and positive signal (f). All images were characterized using Image J. Quantitative comparisons of the ROS levels depict ROS level reductions of $67.95 \%$ in GNC-pretreated worms $(\mathrm{g}, \mathrm{h}) .{ }^{* * *} p \leq 0.001$.

Next, we investigated whether GNC treatment decreased intracellular ROS levels under Cr (VI)-induced stress conditions. As presented in Figure 3, GNC $(3.94 \mathrm{mg} / \mathrm{mL})$ significantly reduced total ROS levels $(67.95 \%, p<0.0001)$ compared with the vehicle control, suggesting that GNC has ROS-scavenging ability.

3.3. GNC Increased Oxidative Stress Resistance in C. elegans via DAF-16/FOXO. Many genes and pathways are associated with oxidative stress responses and longevity (e.g., the IIS, dietary restriction (DR), and germline signaling pathways). To determine the genes/pathways involved in GNC-mediated increases in oxidative stress resistance, we performed $\mathrm{Cr}$ (VI)-induced oxidative stress tests using several characterized mutant strains. Our results showed that GNC did not enhance stress resistance in daf-16 mutants, indicating that daf-16 function is essential for the observed GNC-mediated increase in oxidative stress resistance. In contrast, significant lifespan enhancement was maintained in other mutant strains, daf-2 (e1370), age-1 (hx546), nuo-6 (qm200), isp-1 (qm150), eat-2 (ad465), rsks-1 (ok1255), and glp-1 (e2144)
(Figure 4), suggesting that the protective effect of GNC is independent of the function of these genes.

To further confirm that GNC enhances oxidative stress resistance in $C$. elegans by regulating daf-16-encoded activity, we examined the effect of GNC on the following daf-16targeted genes: sod-3, mtl-1, ctl-2, hsp-12.6, and hsp-16.2 $[25,26]$. Expression levels of sod-3, mtl-1, hsp-12.6, and hsp16.2 were significantly increased, and the upregulation of ctl2 exhibited a tendency toward significance (1.6-fold, $p=$ 0.068). These results suggest that GNC increases oxidative stress resistance via daf-16 (Figure 5).

\section{Discussion}

Traditional Chinese medicine is widely used to relieve declining functions related to aging $[2,27]$. Several studies have shown that GNC improves learning and memory, delays skin aging, and confers resistance to stress conditions [5, 6]. However, the mechanism through which GNC exerts these actions remains unclear. The current study employed C. elegans as an in vivo model to investigate the protective 


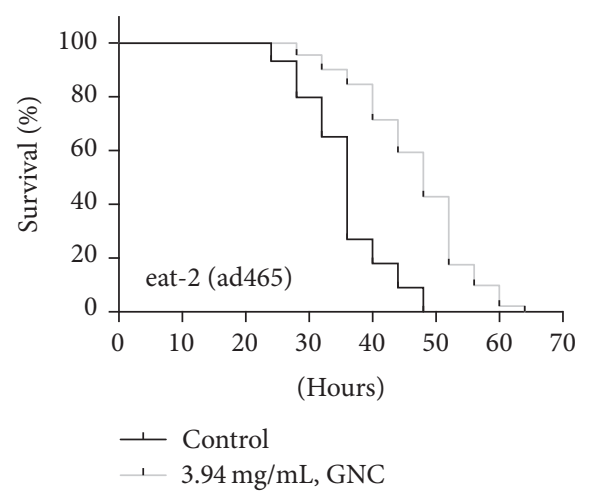

(a)

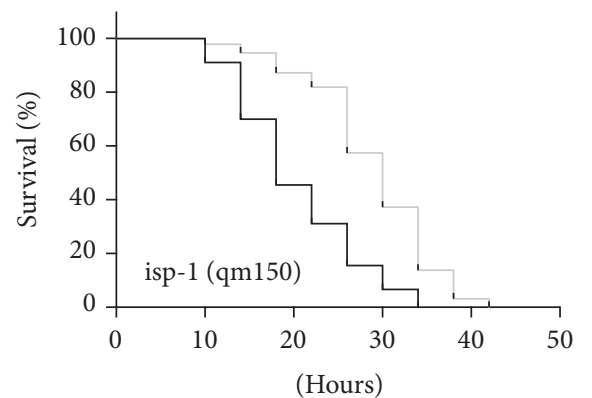

$\longrightarrow$ Control

I $3.94 \mathrm{mg} / \mathrm{mL}$, GNC

(c)

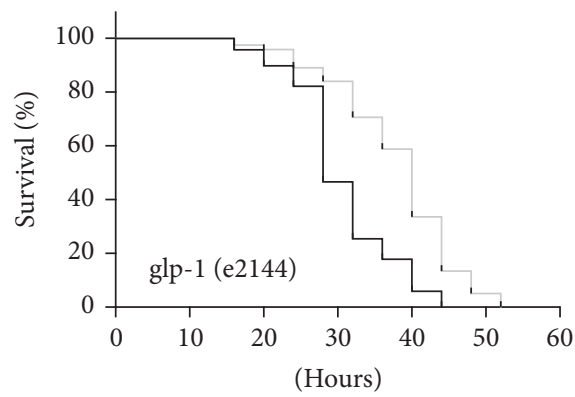

- Control

$\rightarrow 3.94 \mathrm{mg} / \mathrm{mL}, \mathrm{GNC}$

(e)

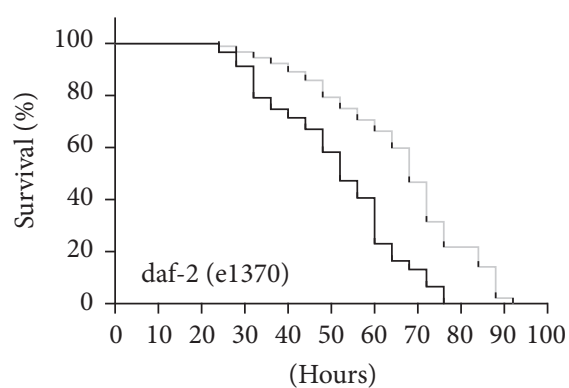

- Control

, $3.94 \mathrm{mg} / \mathrm{mL}, \mathrm{GNC}$

(g)

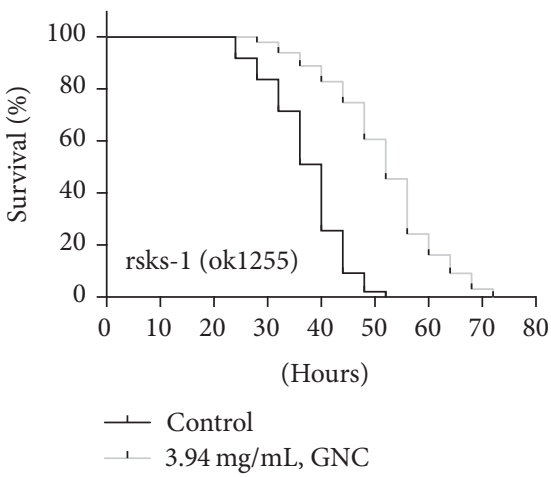

(b)

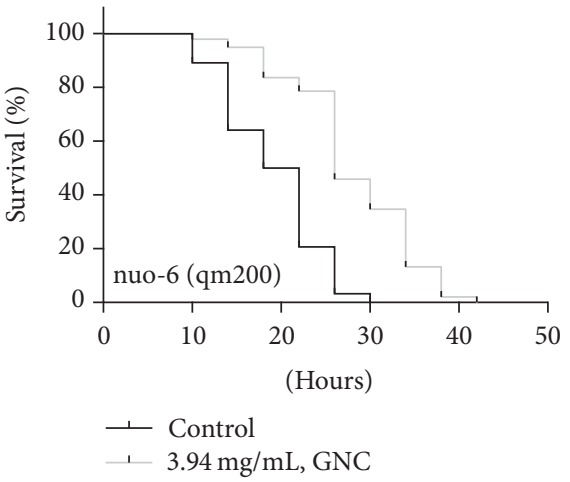

(d)

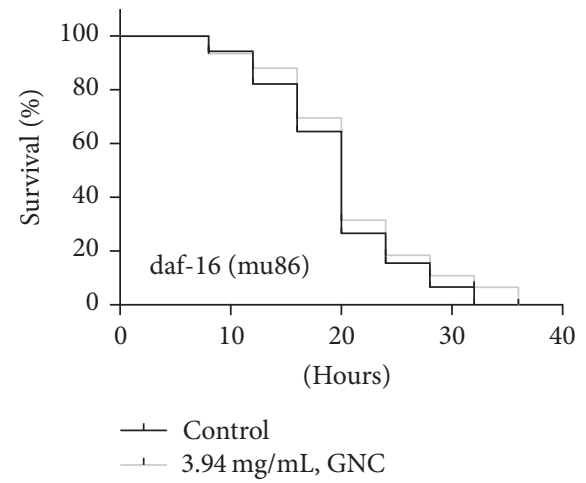

(f)

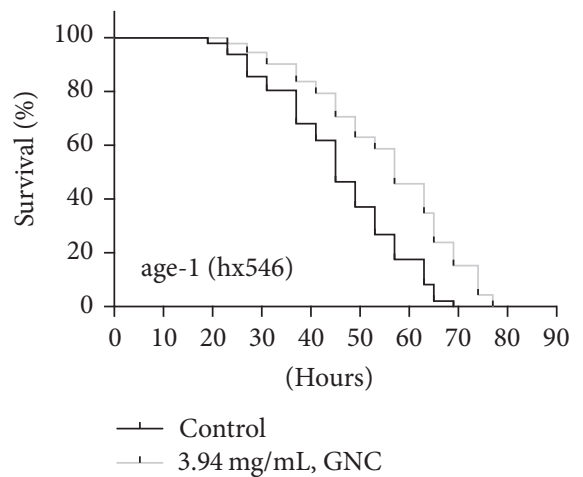

(h)

FIGURE 4: The effect of GNC on the oxidative stress resistance of characterized mutant strains. Notes: survival curves of mutants under Cr (VI)-induced oxidative stress conditions: (a) eat-2 (ad465); (b) rsks-1 (ok1255); (c) isp-1 (qm150); (d) nuo-6 (qm200); (e) glp-1 (e2144); (f) daf-16 (mu86); (g) daf-2 (e1370); and (h) age-1 (hx546), pretreated with an $\mathrm{H}_{2} \mathrm{O}$ control (black) or $3.94 \mathrm{mg} / \mathrm{mL}$ of GNC (grey). The protective effect of GNC depended on the function of daf-16 but was independent of the eat-2, rsks-1, isp-1, nuo-6, glp-1, daf-2, and age-1 genes. 


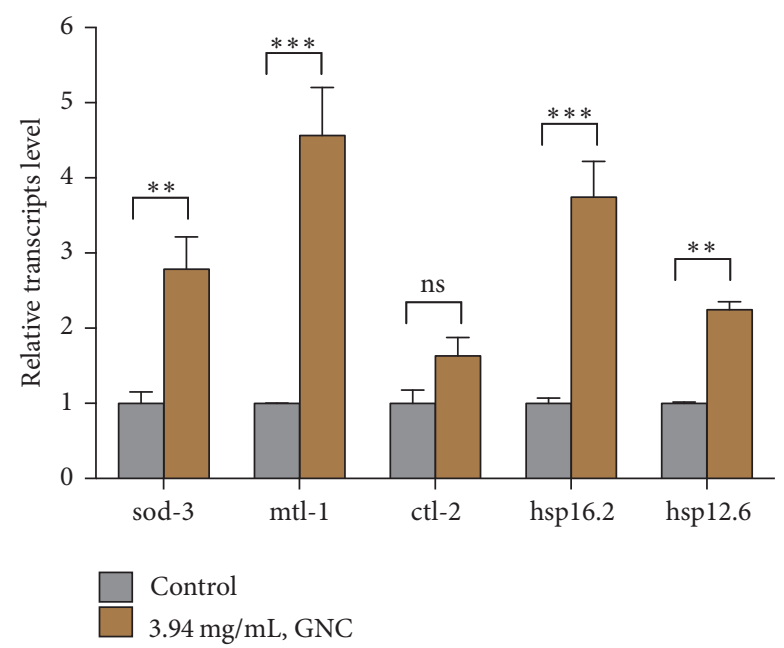

FIGURE 5: Quantification of mRNA expression levels of daf-16targeted genes in GNC-treated worms. Notes: GNC $(3.94 \mathrm{mg} / \mathrm{mL})$ treatment affected relative transcript levels of the genes sod-3, mtl1, ctl-2, hsp-12.6, and hsp-16.2. $\beta$-Actin (act-4) was used as an endogenous control, and the expression levels were determined by real-time PCR using the $2^{-\Delta \Delta C T}$ method. The data are displayed as the means \pm SEMs. ${ }^{* *} p \leq 0.01 ;{ }^{* * *} p \leq 0.001$; ns: not significant.

potential and mechanisms of GNC. We observed significant lifespan increases of $21.0 \%, 31.3 \%$, and $26.3 \%$ at $1.97,3.94$, and $11.82 \mathrm{mg} / \mathrm{mL}$ of GNC, respectively. These findings indicate that GNC extends the lifespan of C. elegans in a dosedependent manner.

As previously reported, oxidative stress damage increases while resistance to cellular stress declines with age [28]. The enhanced lifespan of $C$. elegans has been correlated with improved oxidative stress resistance, and multiple studies have shown that lifespan-extending interventions affect resistance to oxidative stress $[29,30]$. Accordingly, we performed an oxidative stress resistance assay, and our findings showed that the survival time of wild-type worms under oxidative stress conditions was significantly increased following GNC treatment. Moreover, we found that GNC reduced ROS levels by $67.95 \%$, which suggests that GNC has antioxidative capacities due to its ROS-scavenging ability and further indicates that lifespan is positively correlated with resistance to oxidative stress. Although the exact role that oxidative stress plays in the aging process is unknown, it clearly is crucial. Furthermore, oxidative stress damages cellular structures and results in premature cell death. Increasing evidence shows that oxidative stress is involved in various diseases including neurological and cardiovascular diseases as well as metabolic disorders [14]. Previous research demonstrated that GNC has a protective function in oxidative-associated disorders such as skin aging in rat models [6], and given its antioxidative activity in C. elegans, we hypothesized that antioxidative activity is a primary mechanism of the effects of GNC.

Insulin/IGF-1 signaling, germline signaling, DR, and mitochondrial respiration have all been extensively studied, and these pathways have conserved roles not only in regulating the aging process but also in controlling other functions such as stress resistance, metabolism, and reproduction [31]. To determine which pathways are involved in the antioxidative activity of GNC, we examined its effect on nematode strains with mutations in these pathways.

DR increases stress resistance, and previous studies have shown that it induces defensive mechanisms involved in ROS detoxification [32] and increases resistance to lethal heat stress [33]. The eat-2 (ad465) II mutant is regarded as a DR model with pharyngeal pumping defects. In our study, the average survival time under the oxidative condition of eat- 2 (ad465) II pretreated with GNC was extended by $32 \%(p<$ 0.001), indicating that GNC might not act through a DR mechanism.

mTOR signaling is a highly conserved pathway that regulates cellular stress responses, autophagy, and metabolism. TORC1 mediates phosphorylation of S6 kinase (S6K), thereby playing a major role in mRNA translation [34]; the putative ribosomal protein $\mathrm{S} 6 \mathrm{~K}$ is encoded by rsks-1. We tested survival time of mutant rsks-1 (ok1255) worms under oxidative stress and found that GNC increased antioxidative activity in rsks-1 mutants by up to $39 \%(p<0.001)$, which suggests that GNC does not act on the mTOR signaling pathway.

Mitochondrial respiration plays a central role in energy metabolism; indeed, the ROS produced within mitochondria represent almost $90 \%$ of all cellular ROS produced [35]. To investigate whether GNC increases the antioxidative activity of $C$. elegans via the mitochondrial respiration pathway, we tested the effect of GNC on the mutants isp-1 (qm150) and nuo-6 (qm200), encoding Rieske iron-sulfur protein in complex III and a subunit of the NADH dehydrogenase complex [36], respectively. The results showed that GNC increased average survival times under oxidative conditions in isp-1 and nuo-6 (qm200) worms by $42 \%(p<0.001)$ and $47 \%(p<0.001)$, respectively. These results suggest that the antioxidative activity of GNC does not depend on the isp-1 and nuo- 6 genes.

In addition to propagation, the reproductive system of C. elegans is involved in regulating metabolism, autophagy, stress resistance, and longevity [37]. We performed an oxidative stress resistance assay using the mutant glp-1 (e2144) and found increases in antioxidative activity of $24 \%(p<0.001)$ by GNC, suggesting that GNC functions independently of the germline signaling pathway.

The FOXO transcription factor daf-16 plays a central role in regulating the cellular stress response and promoting cellular antioxidant defenses [25]. We tested whether daf-16 is required for the protective effect of GNC, and our results showed that GNC did not increase the survival time of null mutant daf-16 (mu86) worms $(p>0.05)$ under oxidative stress conditions. This finding suggests that the effect of GNC depends on daf-16. To further validate that GNC increased antioxidative activity in worms by regulating daf-16 activity, we measured the expression levels of the following daf-16targeted genes: sod-3, mtl-1, ctl-2, hsp16.2, and hsp12.6. Levels of sod-3, mtl-1, hsp16.2, and hsp12.6 were all significantly increased in worms pretreated with GNC, suggesting that GNC mediates oxidative stress resistance via daf-16. Insulin/IGF-1 signaling, the first pathway discovered to be associated with lifespan, ultimately regulates the forkhead 
transcription factor daf-16 [38]; daf-2, an ortholog of the mammalian insulin and insulin-like growth factor-1 (IGF-1) receptor, phosphorylates age-1, the catalytic subunit of class-I phosphatidylinositol 3-kinase (PI3K), which normally generates PIP3, thereby preventing daf-16 from activating via stimulation of Akt (AKT-1/2) [39]. To investigate whether GNC interacts with molecules in the insulin/IGF-1 signaling pathway to increase antioxidative functions and daf-16 activity, an oxidative stress resistance assay was conducted using mutant daf-2 (e1370) and age-1 (hx546) strains. The results showed that GNC increased average survival time in daf-2 (e1370) and age-1 (hx546) by $28 \%(p<0.001)$ and $21 \%(p<0.001)$, respectively, under oxidative conditions. Thus, GNC induces oxidative stress resistance independent of the insulin/IGF-1 signaling pathway. Taken together, our data suggest that GNC enhances oxidative stress resistance via daf-16/FOXO in C. elegans. Daf-16/FOXO integrates signals from multiple pathways in $C$. elegans, regulating many important biological processes including lifespan, development, metabolism, and stress resistance. However, previous research has indicated that daf-16 does not function alone; rather, it requires other molecules for its activity. c-Jun N-terminal kinase (JNK), a stress-activated MAPK family member, phosphorylates daf-16 in vitro, acting in parallel with the insulin-signaling pathway to regulate daf-16 directly. Similarly, CST-1, the C. elegans homolog of the mammalian Ste20-like kinase MST1, regulates daf-16 in response to oxidative stress [40]. When activated, daf-16 relocates to the nucleus and stimulates transcription of genes that encode antioxidant proteins including antioxidant enzymes such as superoxide dismutase (sod-3), catalases (ctl-1, ctl-2), and metallothionein (mtl-1) [25]. Moreover, together with HSF-1, daf-16 promotes expression of the small heat-shock protein genes hsp-16.1, hsp-16.49, hsp-12.6, and sip-1 [41]. Daf-16 also regulates transcription of numerous other genes to protect worms from damage due to heat stress, oxidative stress, and pathogens, thereby increasing survival under harsh conditions. Furthermore, daf-16 is involved in posttranslational modifications and interactions with coregulators that constitute important transcriptional regulatory mechanisms [25].

A wide variety of modern aging-related diseases such as cancer, diabetes, and neuronal degeneration diseases have become significant threats to human health, and importantly, susceptibility to these diseases increases with age [42]. As opposed to more conventional, single-disease treatment approaches, application of TCM formulas to interfere with the aging process to delay the onset of age-related diseases has begun to receive more attention. However, research on the nature of TCM formulas is rather difficult due to the composition complexity. The results of the present study strongly indicate that the GNC formula enhances resistance to oxidative stress and extends lifespan. It will be interesting and important to determine the ingredient(s) responsible for these effects of GNC in future studies.

\section{Conclusions}

In summary, the current study reports that GNC, a TCM, increases resistance to oxidative stress and promotes longevity in $C$. elegans. These findings are consistent with those of recent studies showing that GNC protects against oxidative stress and has beneficial effects against age-related diseases [3-6]. The antioxidative stress activity of GNC depends on daf-16/FOXO and selective activation of its downstream targets associated with oxidative stress defenses. These findings suggest that GNC acts as an antioxidative agent, and regulation by daf- 16 partially explains the therapeutic effects of GNC. Therefore, GNC shows therapeutic potential for preventing oxidative stress-related diseases such as Alzheimer's disease. Additional tests should be conducted using more complex animals. In addition, because TCM has an expansive pharmacopeia with an array of biological activities that are varied and complex, our use of C. elegans as an animal model to investigate the mechanism of GNC provides a novel method for screening the biological mechanisms of TCMs.

\section{Conflicts of Interest}

The authors declare that there are no conflicts of interest regarding the publication of this paper.

\section{Acknowledgments}

This study was supported by the National Natural Science Foundation of China (no. 81273956) and the Specialized Research Fund for the Doctoral Program of Higher Education (no. 20130071110062). The study was conducted at Shanghai Research Center for Model Organisms, Shanghai 201203, China. The authors are grateful to Professor Jian Fei, Dr. Ping Yang, and Dr. Zhuanbin Wu for their technical support and assistance.

\section{References}

[1] L. X. Cao, Q. Z. Mao, and J. Yu, "Clinical research of Gengnianchun in the treatment of menopausal syndromes," Shanghai Journal of Traditional Chinese Medicine, vol. 2, pp. 33-35, 1997 (Chinese).

[2] F. G. Zhao, W. J. Wang, W. J. Zhou, and D. J. Li, "Effect of Gengnianchun recipe on learning memory function and hippocampal cholinergic system in ovariectomized rats," Chinese Journal of Integrated Traditional and Western Medicine, vol. 3, pp. 234-237, 2008 (Chinese).

[3] J. Li, W. Wang, D. Li, and W. Zhou, "Gengnianchun recipe inhibits apoptosis of pheochromocytoma cells from betaamyloid 25-35 insult, better than monotherapies and their compounds," Neural Regeneration Research, vol. 6, no. 36, pp. 2815-2821, 2011.

[4] S. Z. Bu, J. Yu, Y. L. Zhang et al., "Effect of 'Geng Nian jian' on esrogen receptor mRNA, substance $\mathrm{P}$ and Beta- endorphin in hypothalamus of aging female rats," Biology of Reproduction, vol. 54, supplement 1, p. 151, 1996.

[5] Y.-Q. Rao, J. Li, and W.-J. Wang, "Effects of gengnianchun on learning and memory ability, neurotransmitter, cytokines, and leptin in ovariectomized rats," International Journal of Clinical and Experimental Medicine, vol. 8, no. 6, pp. 8648-8660, 2015. 
[6] Y. Q. Yuan, Effects and Mechanism of Gengnianchun Formula on Skin Aging in Ovariectmized SD Rats, Fudan University, Shanghai, China, 2008.

[7] T. Beckhauser, J. Francis-Oliveira, and R. De Pasquale, "Reactive oxygen species: physiological and physiopathological effects on synaptic plasticity," Journal of Experimental Neuroscience, vol. 10, supplement 1, pp. 23-48, 2016.

[8] H. N. Siti, Y. Kamisah, and J. Kamsiah, "The role of oxidative stress, antioxidants and vascular inflammation in cardiovascular disease (a review)," Vascular Pharmacology, vol. 71, pp. 4056, 2015.

[9] G. Perry, A. D. Cash, and M. A. Smith, "Alzheimer disease and oxidative stress," Journal of Biomedicine and Biotechnology, vol. 2, no. 3, pp. 120-123, 2002.

[10] G. S. Gaki and A. G. Papavassiliou, "Oxidative stress-induced signaling pathways implicated in the pathogenesis of Parkinson's disease," NeuroMolecular Medicine, vol. 16, no. 2, pp. 217230, 2014.

[11] J. Yang, X. Yang, H. Zou, and M. Li, "Oxidative stress and Treg and Th17 dysfunction in systemic lupus erythematosus," Oxidative Medicine and Cellular Longevity, vol. 2016, Article ID 2526174, 9 pages, 2016.

[12] R. K. Gupta, A. K. Patel, N. Shah et al., "Oxidative stress and antioxidants in disease and cancer: a review," Asian Pacific Journal of Cancer Prevention, vol. 15, no. 11, pp. 4405-4409, 2014.

[13] D. Ortuño-Sahagún, M. Pallàs, and A. E. Rojas-Mayorquín, "Oxidative stress in aging: advances in proteomic approaches," Oxidative Medicine and Cellular Longevity, vol. 2014, Article ID 573208, 2014.

[14] K. Kandola, A. Bowman, and M. A. Birch-Machin, "Oxidative stress-a key emerging impact factor in health, ageing, lifestyle and aesthetics," International Journal of Cosmetic Science, vol. 37, supplement 2, pp. 1-8, 2015.

[15] A. E. Sluder and R. Baumeister, "From genes to drugs: target validation in Caenorhabditis elegans," Drug Discovery Today: Technologies, vol. 1, no. 2, pp. 171-177, 2004.

[16] R. Baumeister, E. Schaffitzel, and M. Hertweck, "Endocrine signaling in Caenorhabditis elegans controls stress response and longevity," Journal of Endocrinology, vol. 190, no. 2, pp. 191-202, 2006.

[17] M. Rodriguez, L. Basten Snoek, M. De Bono, and J. E. Kammenga, "Worms under stress: C. elegans stress response and its relevance to complex human disease and aging," Trends in Genetics, vol. 29, no. 6, pp. 367-374, 2013.

[18] E. Moreno-Arriola, N. Cárdenas-Rodríguez, E. CoballaseUrrutia, J. Pedraza-Chaverri, L. Carmona-Aparicio, and D. Ortega-Cuellar, "Caenorhabditis elegans: a useful model for studying metabolic disorders in which oxidative stress is a contributing factor," Oxidative Medicine and Cellular Longevity, vol. 2014, Article ID 705253, 9 pages, 2014.

[19] A. Rousakis, A. Vlassis, A. Vlanti, S. Patera, G. Thireos, and P. Syntichaki, "The general control nonderepressible-2 kinase mediates stress response and longevity induced by target of rapamycin inactivation in Caenorhabditis elegans," Aging Cell, vol. 12, no. 5, pp. 742-751, 2013.

[20] W. Yang and S. Hekimi, "A mitochondrial superoxide signal triggers increased longevity in Caenorhabditis elegans," PLoS Biology, vol. 8, no. 12, Article ID e1000556, 2010.

[21] J. S. Sangha, X. Sun, O. S. D. Wally et al., "Liuwei Dihuang (LWDH), a Traditional Chinese Medicinal Formula, protects against $\beta$-amyloid toxicity in transgenic Caenorhabditis elegans," PLoS ONE, vol. 7, no. 8, Article ID e43990, 2012.
[22] B. Zhu, P. Yang, N. Mammat et al., "Aiweixin, a traditional Uyghur medicinal formula, protects against chromium toxicity in Caenorhabditis elegans," BMC Complementary and Alternative Medicine, vol. 15, article 285, 2015.

[23] J. A. Lewis and J. T. Fleming, "Basic culture methods," in Caenorhabditis elegans: Modern Biological Analysis of an Organism, H. F. Epstein and D. C. Shakes, Eds., pp. 3-29, Academic Press, San Diego, Calif, USA, 1995.

[24] P. Yang, R. Sun, M. Yao, W. Chen, Z. Wang, and J. Fei, "A Cterminal truncated mutation of spr-3 gene extends lifespan in Caenorhabditis elegans," Acta Biochimica et Biophysica Sinica, vol. 45, no. 7, pp. 540-548, 2013.

[25] A. Mukhopadhyay, S. W. Oh, and H. A. Tissenbaum, "Worming pathways to and from DAF-16/FOXO," Experimental Gerontology, vol. 41, no. 10, pp. 928-934, 2006.

[26] C. T. Murphy, S. A. McCarroll, C. I. Bargmann et al., "Genes that act downstream of DAF-16 to influence the lifespan of Caenorhabditis elegans," Nature, vol. 424, no. 6946, pp. 277284, 2003.

[27] L. Zhang and W.-J. Wang, "Research advances of traditional Chinese medicine in delaying skin aging," Journal of Chinese Integrative Medicine, vol. 7, no. 3, pp. 276-279, 2009.

[28] J. M. Van Raamsdonk and S. Hekimi, "Reactive oxygen species and aging in Caenorhabditis elegans: causal or casual relationship?" Antioxidants and Redox Signaling, vol. 13, no. 12, pp. 19111953, 2010.

[29] S. Havermann, H.-U. Humpf, and W. Wätjen, "Baicalein modulates stress-resistance and life span in C. elegans via SKN-1 but not DAF-16," Fitoterapia, vol. 113, pp. 123-127, 2016.

[30] T. Ogawa, Y. Kodera, D. Hirata, T. K. Blackwell, and M. Mizunuma, "Natural thioallyl compounds increase oxidative stress resistance and lifespan in Caenorhabditis elegans by modulating SKN-1/Nrf," Scientific Reports, vol. 6, Article ID 21611, 2016

[31] S. Wolff and A. Dillin, "The trifecta of aging in Caenorhabditis elegans," Experimental Gerontology, vol. 41, no. 10, pp. 894-903, 2006.

[32] M. Ristow and S. Schmeisser, "Extending life span by increasing oxidative stress," Free Radical Biology and Medicine, vol. 51, no. 2, pp. 327-336, 2011.

[33] M. J. Muñoz, "Longevity and heat stress regulation in Caenorhabditis elegans," Mechanisms of Ageing and Development, vol. 124, no. 1, pp. 43-48, 2003.

[34] P. Kapahi, D. Chen, A. N. Rogers et al., "With TOR, less is more: a key role for the conserved nutrient-sensing TOR pathway in aging," Cell Metabolism, vol. 11, no. 6, pp. 453-465, 2010.

[35] I. Bratic and A. Trifunovic, "Mitochondrial energy metabolism and ageing," Biochimica et Biophysica Acta, vol. 1797, no. 6-7, pp. 961-967, 2010.

[36] A. B. Hwang and S.-J. Lee, "Regulation of life span by mitochondrial respiration: The HIF-1 and ROS connection," Aging, vol. 3, no. 3, pp. 304-310, 2011.

[37] A. Antebi, "Regulation of longevity by the reproductive system," Experimental Gerontology, vol. 48, no. 7, pp. 596-602, 2013.

[38] C. Kenyon, "The first long-lived mutants: discovery of the insulin/IGF-1 pathway for ageing," Philosophical Transactions of the Royal Society of London Series B: Biological Sciences, vol. 366, no. 1561, pp. 9-16, 2011.

[39] W. H. Biggs III, J. Meisenhelder, T. Hunter, W. K. Cavenee, and K. C. Arden, "Protein kinase B/Akt-mediated phosphorylation promotes nuclear exclusion of the winged helix transcription 
factor FKHR1," Proceedings of the National Academy of Sciences of the United States of America, vol. 96, no. 13, pp. 7421-7426, 1999.

[40] J. N. Landis and C. T. Murphy, "Integration of diverse inputs in the regulation of Caenorhabditis elegans DAF-16/FOXO," Developmental Dynamics, vol. 239, no. 5, pp. 1405-1412, 2010.

[41] A.-L. Hsu, C. T. Murphy, and C. Kenyon, "Regulation of aging and age-related disease by DAF-16 and heat-shock factor," Science, vol. 300, no. 5622, pp. 1142-1145, 2003.

[42] C. Kenyon, "The plasticity of aging: insights from long-lived mutants," Cell, vol. 120, no. 4, pp. 449-460, 2005. 


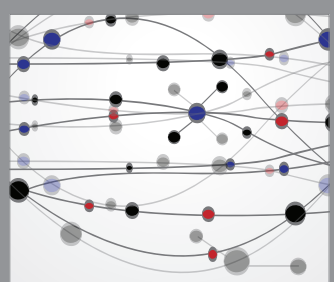

The Scientific World Journal
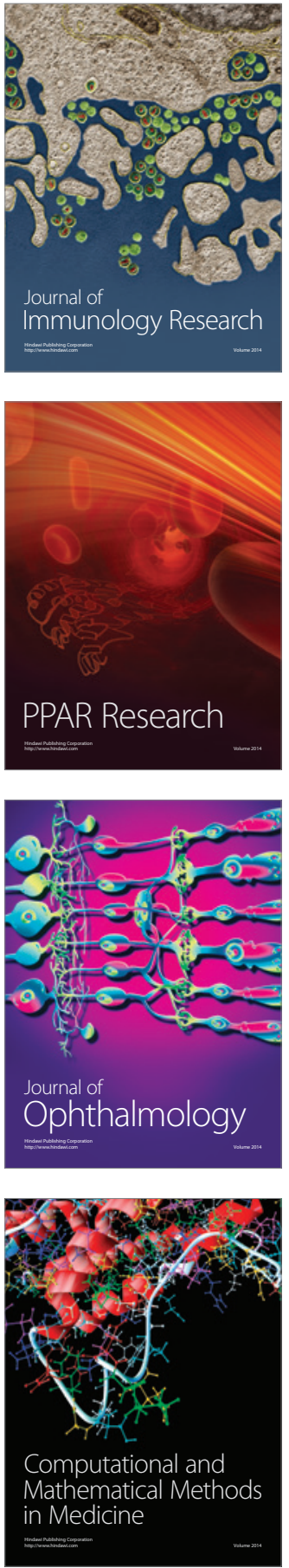

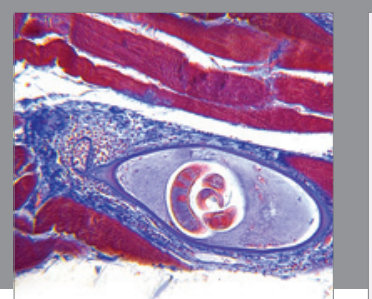

Gastroenterology Research and Practice
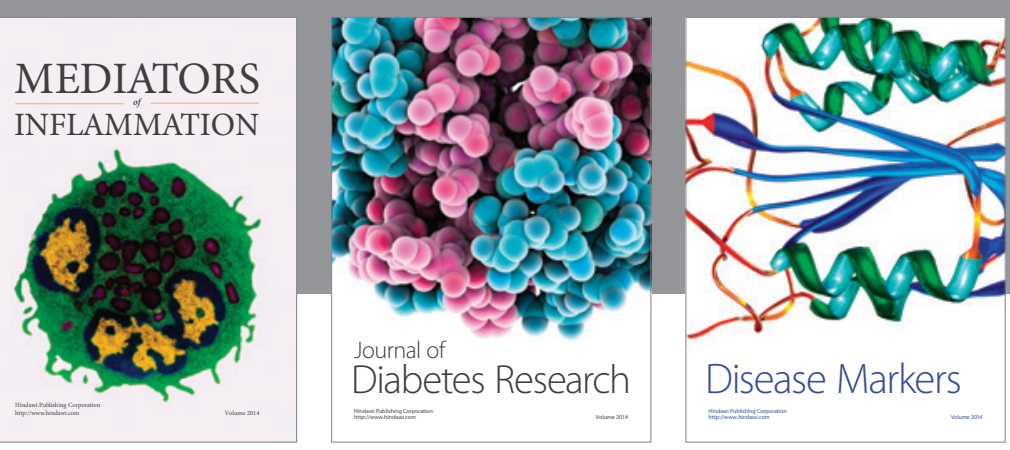

Disease Markers

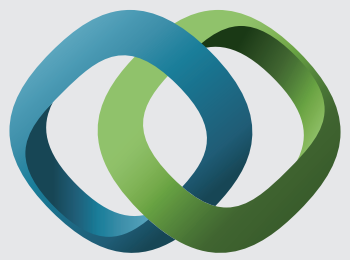

\section{Hindawi}

Submit your manuscripts at

https://www.hindawi.com
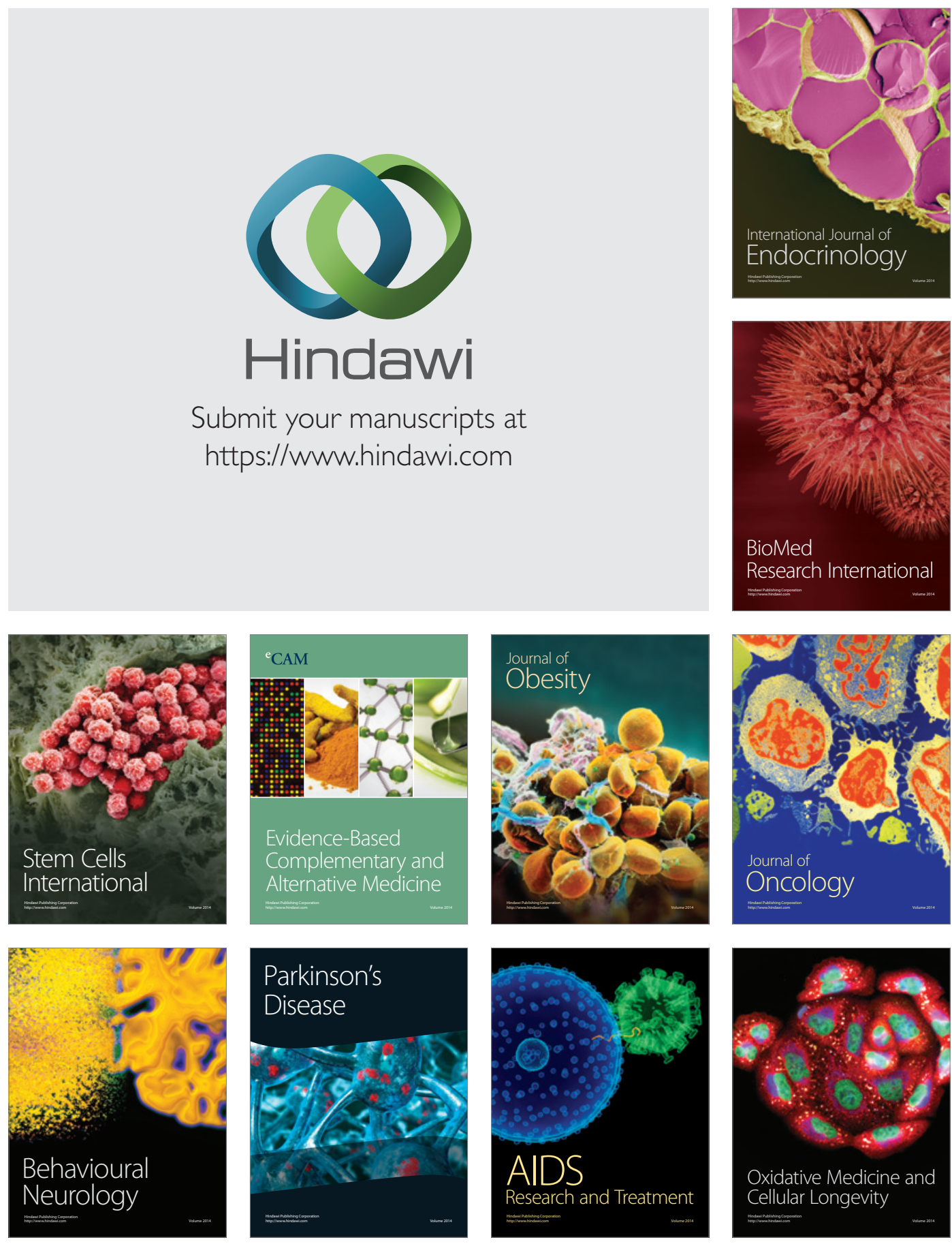